\title{
Biodiversity and Phytosociological Analysis of Plants in Wadi Al-Quf Nursery Reserve North - Western of Hebron City in Palestine
}

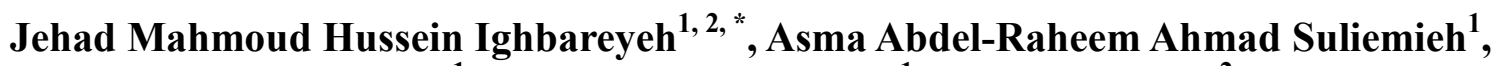 \\ Azhar Mousa Abu Ayash ${ }^{1}$, Maen Nimer Sheqwara ${ }^{1}$, Ana Cano Ortiz ${ }^{2}$, Eusebio Cano Carmona ${ }^{2}$ \\ ${ }^{1}$ Department of Plant Production and Protection, Faculty of Agriculture, Al-Quds Open University, Hebron, Palestine \\ ${ }^{2}$ Department of Animal and Plant Biology and Ecology, Faculty of Experimental Sciences, University of Jaen, Jaen, Spain
}

Email address:

jehadighbareyeh@hotmail.com (J. M. H. Ighbareyeh)

${ }^{*}$ Corresponding author

\section{To cite this article:}

Jehad Mahmoud Hussein Ighbareyeh, Asma Abdel-Raheem Ahmad Suliemieh, Azhar Mousa Abu Ayash, Maen Nimer Sheqwara, Ana Cano Ortiz, Eusebio Cano Carmona. Biodiversity and Phytosociological Analysis of Plants in Wadi Al-Quf Nursery Reserve North - Western of Hebron City in Palestine. Journal of Plant Sciences. Vol. 9, No. 1, 2021, pp. 13-24. doi:

Received: July 17, 2020; Accepted: August 3, 2020; Published: March 4, 2021

\begin{abstract}
This paper presents a vegetation study of the Wadi Al-Quf Nursery Reserve territories of the west-north of Hebron-Palestine. These site is a very important at a local level of species of plant and flora with a high endemicity average. The floristic analysis revealed the existence of 82 species, of which 16 (19.51\%) are endemic and ninety plots of vegetation distributed in this area. The phytosociological approach and analysis are based on the Braun- Blanquet methodology 1979, and used classification of the land for Salvador Rivas Martinez to analysis of the physical factors of the bioclimate and climate. However, Wadi Al-Quf Nursery Reserve, is located at the western of Hebron area and belong to infra Mediterranean to mesoMediterranean thermotype, this area has a characteristic arid, dry and very little of sub-humid and located between Mediterranean basin, Negev, desert Sinai and Red Sea region. We took 300 samples of different species plants from Wadi AlQuf Nursery Reserve. In the statistical treatment we obtained two large groups in the cluster; group (A), representing forests, copses and high shrub lands influenced by climate (climatophilous); and group (B), representing pino copses which are influenced by climatophilous. We have three associations limited as Pistacio lentisci-Quercetum lokii Ighbareyeh J. M. H., A. A. Suliemieh, A. Cano-Ortiz \& E. Cano nova. hoc loco., Ceratonio siliquae-Quercetum callipinii ass. nova. and Pino halepensis-Cupressetum sempervirentis ass. nova., with a three alliance as Pistachio- Quercion lokii, Ceratonio siliquae Quercion calliprinae and Pino halepensis-Cupression sempervirentis.
\end{abstract}

Keywords: Palestine, Wadi Al-Quf, Biology, Flora, Phytosociology and Plant Communities

\section{Introduction}

Palestine's particular geographic location, in conjunction with a series of environmental, bioclimatic factors, soil type, makes this a very fertile land [1]. Climate and bio-climate were played an important role in influence on biodiversity [2], plant communities [2-4], biology process and biological resources [5]. Flora of Palestine contains 149 species of endemic ( $6 \%$ of all plants), $43 \%$ of which are common, $27.5 \%$ rare and $25.6 \%$ very rare. The Leguminaceae family, for example, has 268 species, including 21 species of which endemic, whereas
23 species of Iridaceae, eight of which are endemic [6], but today there are more than 155 species of endemics [2, 5]. However, it is the meeting ground for plant species that originate from various regions of the world, such as Western Europe, Central Asia and East Africa. They are characterized by a wide range of wildlife resources and represent a rich base of flora and fauna in which natural organisms are formed by an estimated 2,483 species of plants living in Palestine [7], also Palestine, located in the Mediterranean Basin, is considered the hot spot of the world's biodiversity that should be conserved [8,9], these plants are ecologically adapted in different parts of 
Palestine, extending from the extreme north to south, especially in the Negev Desert, Sinai and the Mediterranean Sea to the west, and the Dead Sea to the east $[10,11]$. Flora Palestine is rich in important economic plants and includes vegetables, crops fields, fruit trees and the plants are use in the medical, and the recent studies there are more than 2,750 species of plants, including 138 families of Palestinian plants [12]. In Palestine (Palestinian territories occupied in 1967), about 2780 plant taxa were recorded as native or endemic, from the native taxa, 162 taxa were recorded as endemics [1, 2], were showed 1881 deference species of plant, which of them 53 species endemic rare in the fourth areas of Palestine an area of $1145 \mathrm{~km}^{2}$ as Trifolium palaestinum Boiss., Paronychia palaestina Eig., Suaeda philistaeum Zohary and Trifolium philistaeum.

In general, the forests of Hebron governorate are distributed throughout the governorate and are characterized by their Mediterranean environmental system, there are almost 14,949 dunms of forested areas in the Hebron, it accounts for $22 \%$ of Palestine's forests. The climate tends to be sub-humid to dry from west to east [13-16], providing suitable environments for the growth of most plant species. Most of the forests of Hebron are located on fertile soils (Terra Rosa, Brown Ruinsenas and Pali Rindzinas) and in areas with favorable climatic, bioclimatic conditions for agriculture [17, 18].

During the past decade and tenth years ago, several studies have analyzed the phytosociological and flora [5, 19-25] and gathering of wild edible plants in specific countries in the Mediterranean area including, Italy $[26,27]$ and Spain $[28$, 29] and others regions [30].

The main aims of the present study are to contribute to the knowledge of plant taxa, biodiversity, flora, and to phytosociological with others characteristics biologicals and ecologically of plant in Wadi Al-Quf Nursery Reserve, Hebron of Palestine.

\section{Methods}

Due to the lack of significant meteorological data, we selected a sampling area in the region of Wadi Al-Quf in Hebron, in which inventories were taken of 300 woody plants in this location (Figure 1). Wadi Al-Quf Forest or Nursery Reserve and a little of scrub-lands were sampled to obtain biological, environmental indicators and vegetation for ombrotype \& thermotype. The inventories were made following Braun-Blanquet (1979) [31], and the flora of Palestine, Syria, Lebanon, Jordan, and Sinai desert were used for the floristic study. Addition to our study deals with the vegetation of the western Hebron, located to the east of the occupied Palestinian coast as Beit Jibreen, Al Manshiyya Iraq, Ajur, Dawayima and the Mediterranean coast, they are therefore part of this nearby plant environment. Besides, we transformed the Braun-Blanquet species abundance dominance values into Van der Maarel (1979) [32]. We found only a few phytosociological works on areas in Palestine [3], Egypt which have no relation with our communities [33, 34], and others of an ecological nature [35]. The vegetation was interpreted according to several methodological works as [36-40].

\subsection{Study Area}

Wadi Al-Quf Nursery or Forest Reserve (WANR) is located $6 \mathrm{~km}$ north-west of Hebron, it is the largest green area in the West Bank, covering more than 4,600 dunums planted (which of 2500 dunums with Palestinian) (Figure 1), rises $450-900 \mathrm{~m}$ above sea level and between longitudes $35^{\circ}$ $02^{\prime}$ east and latitudes $31^{\circ} 34^{\prime}$ north (Table 1), there are also five springs and a historic cave in the Reserve (Figure 2). Wadi Al-Quf Nursery Reserve is also located on a series of mountain hills and a large valley, extending from the highest central and eastern heights of the city of Hebron towards the lowlands in the semi-coastal area. It is worth noting that the Wadi Al-Quf Nursery Reserve was established in the middle of the eighteenth century, i.e. in the Ottoman era, what distinguishes the Wadi Al-Quf Nursery Reserve is that it has maintained its permanence in its green zone and its biodiversity throughout history, from the Ottoman era (before 1870-1927) to the British Mandate (1927-1948) and then the Israeli occupation (1948 to present).

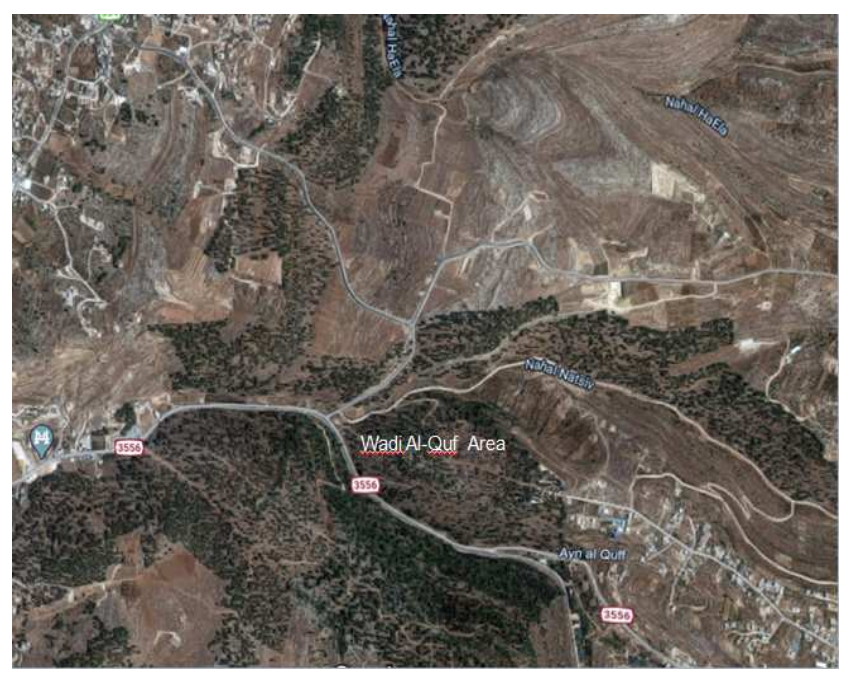

Figure 1. Represents study area, plants sampled in Wadi Al-Guf Nursery Reserve in Hebron of Palestine by satellite.

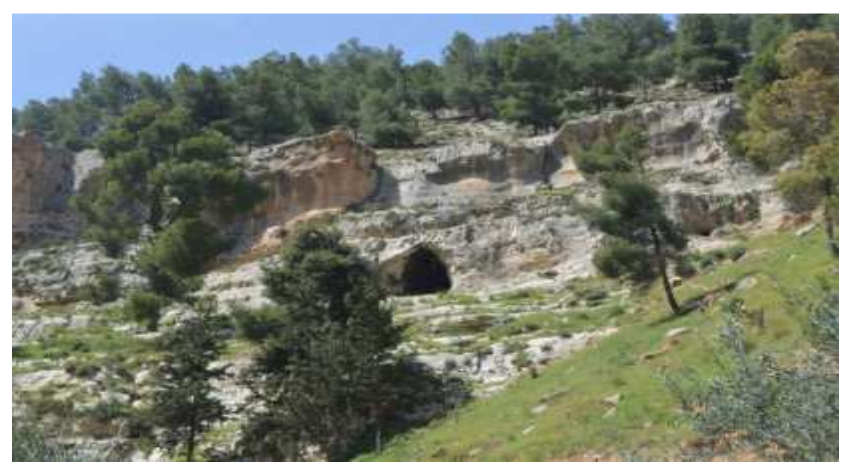

Figure 2. Represents of the famous cave in the Wadi Al-Quf, which has inside Nursery Reserve west of the north of Hebron. 


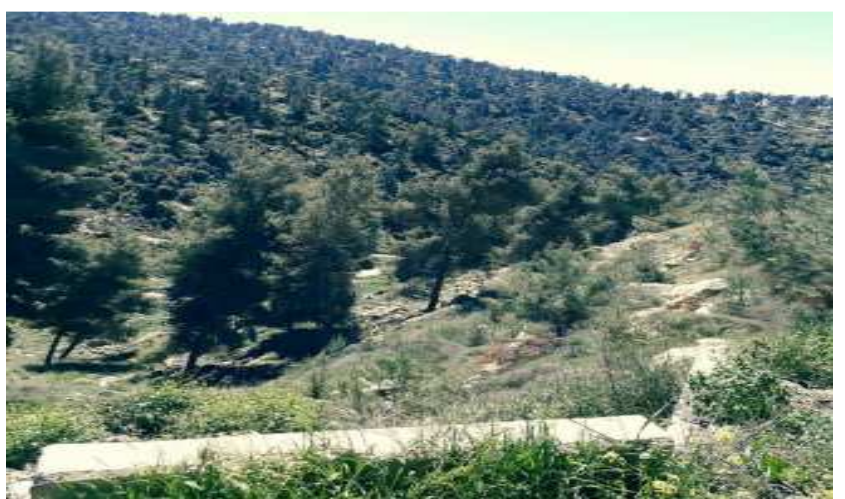

Figure 3. An image representing part of the plants in the Wadi Al-Guf Nursery Reserve.

\subsection{Targeting and Collection of Plant Data}

We took 300 samples of different species plants from Wadi Al-Quf Forest or Nursery Reserve during February to Mayo 2020 to study the plant taxa and communities, phytosociology and flora. The western Hebron has a dry ombroclimate with rainfall rates ranging from $300-600 \mathrm{~mm}$, ombrothermic index records for these one sites are 2.5 as Idna village [20], and the thermotype is infratropical with thermicity index/compensated thermicity index values of $350 / 460$ in Idna village $[5,21]$.

\section{Results}

Statistical Analysis

In this work, bioclimatic, biogeographical and geobotanical analyses are dependent on the contributions of Salvador Rivas-Martínez (2011) [39], (Figure 2), our floral study has adopted suggestions for Liogier (1996) [41]. To determine the groups for statistical purposes, we first created a matrix consisting of 83 rows x 19 columns of relevance (s), which led to the conversion of the phytosociological plant indexes of Braun- Blanquet, $+, 1,2,3,4,5$ to those in Van der Maarel 2, 3, 4, 5, 6, 7. However, we conducted Euclidean Distance Clustering and a Principal Component Analysis, to avoid any loss of information related to proper plant analysis, all sampled studied of plant were taken into account with the groups identified and selected in our statistical analysis as a valid reference basis and Rivas-Martínez [39, 40] (2011, 1999) followed us to create dynamically Phytosociology.

Our statistical analysis, conducted on a clean matrix consisting of 82 rows (species) in 19 columns, resulted in a grouping with two clearly distinct groups (Group A and B). The group A consists of two forest samples group 1 and 2 (G1 and G2). The group G1 (1-14) is dominated by species belonging to the Quercus and Pistacia genus and forests growing in thermomediterranean - Mesomediterranean thermotype and dry to subhumid environments on carbonated substrates as Terra Rosa and Brown Ruinsenas with neutral $\mathrm{pH}$, and this leads us to a proposal, in the Asian regions of the eastern Mediterranean, the association is Group 1. Pistacio lentisci-Quercetum lokii (Table 1: ASL 1._1-14).

Group G2 (2-11) was dominated by Quercus calliprinos Webb., Ceratonia siliqua L. and Quercus look Kotschy forests growing in infra-thermomediterranean thermotype and where the ombrotype is arid-semiarid, and the soil types are Terra Rosa and lithosoils and loess with neutral $\mathrm{pH}$, and this we propose the association of the eastern Mediterranean, is Ceratonio siliquaeQuercetum callipinii (Table 2: ASL 2. 2-11).

Association B is consist of one group forest (9-19) representing by species Cupressus macrocarpaa L., Pinus halepensis L., Pinus Pinea L. Cupressus arizonica Greene., Cupressus macrocarpaa L. with companions Pistacia palaestina Boiss. forests growing a climatophilic community in steppe environments with a semiarid, arid -dry ombroclimate and inframediterranean thermotype. Therefore, we propose the association Pino halepensis-Cupressetum sempervirentis (Table 3: ASL 3. 9-19). The principal components analysis confirms the separation of plant groups (GA as G1, G2 and GB) (Figure 4).

\section{Discussion}

Plant Communities Analysis

The cluster in group GA can be broken down into two plant communities. Subgroup G1 which includes inventories 1-14 (Figure 4) of the cluster that were taken in the Wadi AlGuf Nursery Reserve mountains, in thermotropical environments with a dry -semidry ombrotype on Terra Rossa. We indicated endemic species in this community, of which the following are: Quercus look Kotschy, Pistacia palaestina Boiss., Pyrus syriacus Boiss., which are companions with Rhamnus palaestinus Boiss. species, a with slope is between $10-30 \%$, coverage rate of $75 \%$ of plant, and average height of vegetation (3-6m.) (Table 1).

Table 1. Sampling plots.

\begin{tabular}{lllll}
\hline & Coordinates & Site & Altitude & Biogeographic unit \\
\hline P1 & $31.578854,35.061280$ & Wadi Al-Quf & 580 & Mediterranean basin territories \\
P2 & $31.580999,35.025151$ & Wadi Al-Quf & 460 & Mediterranean basin territories \\
P3 & $31.573196,35.032914$ & Wadi Al-Quf- near of settlement Telem & 600 & Mediterranean basin territories \\
P4 & $31.580999,35.035150$ & Wadi Al-Quf & 660 & Mediterranean basin territories \\
P5 & $31.578854,35.071380$ & Wadi Al-Quf-near of Beit Kahil village & 550 & Mediterranean basin territories \\
P6 & $31.571885,35.076915$ & Wadi Al-Quf & 630 & Mediterranean basin territories \\
P7 & $31.577608,35.026301$ & Wadi Al-Quf-near of Tarqumia village & 660 & Mediterranean basin territories \\
P8 & $31.571885,35.076915$ & Wadi Al-Quf & 550 & Mediterranean basin territories \\
P9 & $31.578854,35.061270$ & Wadi Al-Quf & 900 & Mediterranean basin territories \\
P10 & $31.571885,35.076715$ & Wadi Al-Quf & 450 & Mediterranean basin territories \\
P11 & $31.580999,35.035753$ & Wadi Al-Quf & &
\end{tabular}




\begin{tabular}{lllll}
\hline & Coordinates & Site & Altitude & Biogeographic unit \\
\hline P12 & $31.582071,35.047959$ & Wadi Al-Quf & 520 & Mediterranean basin territories \\
P13 & $31.580999,35.035555$ & Wadi Al-Quf & 490 & Mediterranean basin territories \\
P14 & $31.580999,35.061256$ & Wadi Al-Quf & 850 & Mediterranean basin territories \\
P15 & $31.571885,35.076925$ & Wadi Al-Quf & 750 & Mediterranean basin territories \\
P16 & $31.580999,35.035158$ & Wadi Al-Quf & 700 & Mediterranean basin territories \\
P17 & $31.582071,35.047759$ & Wadi Al-Quf & 520 & Mediterranean basin territories \\
P18 & $31.578854,35.061280$ & Wadi Al-Quf & 550 & Mediterranean basin territories \\
P19 & $31.580999,35.035161$ & Wadi Al-Quf & 460 & Mediterranean basin territories \\
\hline
\end{tabular}

Subgroup G2 includes inventories $2-11$ of the cluster and in (Table 2), the community grows in the areas of the Wadi Al-Quf Nursery Reserve in the dry infra and thermotropical thermotype. This is a dense forest with $16 \%$ endemic plants, of which the following are: Quercus look Kotschy, Pistacia palaestina Boiss. and Rhamnus palaestinus Bois, which are companions with Pistacia palaestina Boiss. species, a with slope is between $15-30 \%$, coverage rate of $70 \%$ of plant, and average height of vegetation (3.5-6m.).

Table 2. Association 1: Pistacio palestinae-Quercetum lokii Ighbareyeh J. M.. H., A. A. Suliemieh, A. Cano-Ortiz \& E. Cano nova. hoc loco. 2014.

\begin{tabular}{|c|c|c|c|c|c|c|c|c|}
\hline Species & & & & & & & & \\
\hline Releve of cluster & 1 & 4 & 12 & 10 & 7 & 14 & $\mathrm{P}$ & $\mathrm{S}$ \\
\hline Surface in $\mathrm{m}^{2} 1=10$ & 250 & 270 & 300 & 290 & 280 & 240 & $\mathrm{R}$ & $\mathrm{T}$ \\
\hline Cover rate $\%$ & 60 & 75 & 75 & 80 & 85 & 75 & $\mathrm{E}$ & A \\
\hline Altitude in $\mathrm{m}$. & 580 & 660 & 520 & 900 & 800 & 850 & S & $\mathrm{T}$ \\
\hline Average height of veg. (m.) & 3 & 4.5 & 5 & 4 & 6 & 3.5 & $\mathrm{~N}$ & $\mathrm{U}$ \\
\hline Slope \% & 25 & 30 & 15 & 20 & 25 & 10 & $\mathrm{C}$ & $\mathrm{S}$ \\
\hline Orientation & $\mathrm{E}$ & $\mathrm{N}$ & $\mathrm{N}$ & W & W & $\mathrm{E}$ & I & \\
\hline Order number & 5 & 2 & 2 & 3 & 1 & 0 & & \\
\hline \multicolumn{9}{|l|}{ Characteristic of association and higher units } \\
\hline Quercus look Kotschy & 4 & 3 & 2 & 2 & 2 & 1 & $\mathrm{~V}$ & $\mathrm{E}$ \\
\hline Pistacia lentiscus L. & 3 & & 2 & 2 & & 1 & Iv & $\mathrm{N}$ \\
\hline Pistacia palaestina Boiss. & 2 & & 2 & & 2 & & III & $\mathrm{E}$ \\
\hline Quercus calliprinos Webb. & 2 & & & & & & I & $\mathrm{N}$ \\
\hline Quercus coccifera $\mathrm{L}$. & & 1 & & & & & I & $\mathrm{N}$ \\
\hline Pyrus syriaca Boiss. & & & & 2 & & & I & $\mathrm{E}$ \\
\hline Quercus inthaburensis L. & & 1 & & 2 & & & III & $\mathrm{N}$ \\
\hline Quercus libani G. Olivie & & 1 & & & & 1 & II & $\mathrm{N}$ \\
\hline \multicolumn{9}{|l|}{ Companions } \\
\hline Rhamnus palaestinus Boiss. & 3 & & 1 & 2 & & 1 & Iv & E \\
\hline Rhamnus alaternus $\mathrm{L}$. & & 1 & & & & & I & $\mathrm{N}$ \\
\hline Zizyphus Spina-christi L. & & 1 & & & & & I & $\mathrm{N}$ \\
\hline Zizyphus Lotus L. & & & + & & & & I & $\mathrm{N}$ \\
\hline Hypecoum pendulum $\mathrm{L}$. & & & & & & & & $\mathrm{N}$ \\
\hline Rhamnus lycioides $\mathrm{L}$ & 1 & & & & & & I & $\mathrm{E}$ \\
\hline Schinus molle L. & & & & + & & & I & $\mathrm{N}$ \\
\hline Pistacia saportae Burnat. & & 1 & & & 1 & & II & $\mathrm{N}$ \\
\hline Rhus coriaria $\mathrm{L}$. & & & + & & & & I & $\mathrm{N}$ \\
\hline Pistacia atlantica Desf. & & & + & & & & I & $\mathrm{N}$ \\
\hline Pinus halepensis Miller & & & 1 & & & & I & $\mathrm{N}$ \\
\hline Pinus Pinea L. & & & & & & 1 & I & $\mathrm{N}$ \\
\hline Pinus canariensis $\mathrm{C}$. Smith & & & & & 2 & & I & $\mathrm{N}$ \\
\hline Pinus brutia Tenore & & & 2 & & & & I & $\mathrm{N}$ \\
\hline Cupressus sempervirens L. var. horizontalis Miller & & & & & 1 & & I & $\mathrm{N}$ \\
\hline Cupressus arizonica Greene & & & 1 & & & & I & $\mathrm{N}$ \\
\hline Thuja occidentalis L. & & 1 & & & 1 & & I & $\mathrm{N}$ \\
\hline Cupressus sempervirens L. & & 1 & & & & 1 & I & $\mathrm{N}$ \\
\hline Crataegus oriana (L) DC. & & & & & & & & $\mathrm{N}$ \\
\hline Crataegus azarolus $\mathrm{L}$. & & & & & & & & $\mathrm{N}$ \\
\hline Amygdalus communis $\mathrm{L}$. & & & & 1 & & & I & $\mathrm{N}$ \\
\hline Prunus dulcis (Miller) D. A. Webb. & 1 & & 1 & & 1 & & III & $\mathrm{N}$ \\
\hline Sarcopoterium spinosum (L.) Spach & & 1 & + & & 1 & & III & $\mathrm{E}$ \\
\hline Ceratonia siliqua $\mathrm{L}$. & & & & 1 & & & I & $\mathrm{N}$ \\
\hline Retama raetam (Forssk) Webb \& Berthel. & & 1 & & & & & I & $\mathrm{N}$ \\
\hline Acacia salicina Lindl. & & & & & 2 & & I & $\mathrm{N}$ \\
\hline Acacia cyanophylla Lindl. & & 1 & & & & & I & $\mathrm{N}$ \\
\hline Sophora japonica L. & & & + & & & & I & $\mathrm{N}$ \\
\hline Calicotome villosa (Poir) Link. & 1 & & & & & & I & $\mathrm{N}$ \\
\hline Spartium junceum L. & 1 & & & & & & I & $\mathrm{N}$ \\
\hline Cersis siliquastrum L. & & 1 & 1 & & & & II & $\mathrm{N}$ \\
\hline
\end{tabular}




\begin{tabular}{|c|c|c|c|c|c|c|c|c|}
\hline Species & & & & & & & & \\
\hline Poinciana gillesii Hook. & & & & 1 & & & I & $\mathrm{N}$ \\
\hline Acacia radiana Savi. & & & & & & & & $\mathrm{N}$ \\
\hline Olea europaea $\mathrm{L}$. & & & & & 1 & & I & $\mathrm{N}$ \\
\hline Phillyria media $\mathrm{L}$. & & & 1 & & 1 & & II & $\mathrm{N}$ \\
\hline Styrex officinalis L. & & & & & & & & $\mathrm{N}$ \\
\hline Laurus nobilis L. & & 1 & & & & & I & $\mathrm{N}$ \\
\hline Balanites aegyptiaca (L.) Delile & 1 & & & 1 & & & II & $\mathrm{N}$ \\
\hline Salix alba $\mathrm{L}$. & & & & & & & & $\mathrm{N}$ \\
\hline Populus alba $\mathrm{L}$. & & & & & & 1 & I & $\mathrm{N}$ \\
\hline Populus nigra $\mathrm{L}$. & & & 1 & & 1 & & I & $\mathrm{N}$ \\
\hline Populus euphratica Oliv. & & & & 1 & & & I & $\mathrm{N}$ \\
\hline Arbatus andrachne L. & + & & 1 & & & & I & $\mathrm{E}$ \\
\hline Platanus orientalis $\mathrm{L}$. & & & & & & & & $\mathrm{N}$ \\
\hline Eucalyptus gomphosephala DC. & & & & & & & & $\mathrm{N}$ \\
\hline Eucalyptus camaldulensis Dehnh & & & & 1 & & & I & $\mathrm{N}$ \\
\hline Ailanthus glandulosa Desf. & & & & 1 & & & I & $\mathrm{N}$ \\
\hline Melia azedarach $\mathrm{L}$. & & & & 1 & & & I & $\mathrm{N}$ \\
\hline Acer obtusifolium $\mathrm{Sm}$. & & & 1 & & & & I & $\mathrm{E}$ \\
\hline Tamarix articulate Vahl. & & & 1 & & & & I & $\mathrm{N}$ \\
\hline Tamarix aphylla $\mathrm{L}$. & & & & & & & I & $\mathrm{N}$ \\
\hline Tamarix jordanis Boiss. & & & + & & & & I & $\mathrm{E}$ \\
\hline Tamarix palestina Bertol. & & & 1 & & & & I & $\mathrm{E}$ \\
\hline Atriplex halimus L. & & 1 & & & & & I & $\mathrm{N}$ \\
\hline Ficus retusa $\mathrm{L}$. & 1 & & & & & & I & $\mathrm{N}$ \\
\hline Ficus sycomorus $\mathrm{L}$. & & & 1 & & 1 & & II & $\mathrm{N}$ \\
\hline Ficus cariaca $\mathrm{L}$. & & & & 2 & & 1 & II & $\mathrm{E}$ \\
\hline Ficus benjamiva $\mathrm{L}$. & & 1 & & & & & I & $\mathrm{N}$ \\
\hline Morus alba L. & & 1 & & & & & I & $\mathrm{N}$ \\
\hline Morus nigara L. & & 1 & & 1 & & & II & $\mathrm{N}$ \\
\hline Spartium junceum L. & & & & 2 & & & I & $\mathrm{N}$ \\
\hline Anagyris foetida $\mathrm{L}$. & & & 1 & & & & & $\mathrm{~N}$ \\
\hline Lycium shawii Roem. \& Schult. & & & & & & & I & $\mathrm{E}$ \\
\hline Lycium barbarum $\mathrm{L}$. & & & 1 & & & & I & $\mathrm{N}$ \\
\hline Lycium europaeum $\mathrm{L}$. & & 1 & & & & & & $\mathrm{~N}$ \\
\hline Artemisia sieberi Besser. & & 1 & & & & & I & $\mathrm{N}$ \\
\hline Juglans regia $\mathrm{L}$. & 1 & & & & & & I & $\mathrm{N}$ \\
\hline Casuarina equesitifolia $\mathrm{L}$. & & & & 1 & & & I & $\mathrm{N}$ \\
\hline Celtis australis $\mathrm{L}$. & & & 1 & & & & I & $\mathrm{N}$ \\
\hline Jacaranda mimosaefolia D. Don & & & & + & & & I & $\mathrm{N}$ \\
\hline Capparis spinosa L. & 1 & & & & & & I & $\mathrm{N}$ \\
\hline Cupressus macrocarpa Hartw. & 1 & & & & & & I & $\mathrm{N}$ \\
\hline Phlomis brachyodon (Boiss.) Zohary & & & & & 1 & & I & $\mathrm{E}$ \\
\hline Phlomis pungens Willd. & & & & & 1 & & I & $\mathrm{N}$ \\
\hline
\end{tabular}

Group B includes inventories 9-19 of the cluster in (Table 3 ), the community or group grows in the areas of the Wadi Al-Quf Nursery Reserve in the dry infra Mediterranean to meso-Mediterranean thermotype. This is a dense forest with $19.51 \%$ endemic plants, of which the following are: Rhamnus palaestinus Bois, Pistacia palaestina Boiss., Pyrus syriaca Boiss. and Arbatus andrachne $\mathrm{L}$ which are companions with Pistacia palaestina Boiss. species, a with slope is between 5$30 \%$, coverage rate of $72 \%$ of plant, and average height of vegetation $(6-10 \mathrm{~m}$.).

Table 3. Association 2: Ceratonio siliquae-Quercetum calliprini ass. nova.

\begin{tabular}{|c|c|c|c|c|c|c|c|c|}
\hline \multicolumn{9}{|l|}{ Species } \\
\hline releve of cluster & 2 & 11 & 8 & 5 & 3 & 6 & $\mathrm{P}$ & $\mathrm{S}$ \\
\hline Surface in $\mathrm{m}^{2} 1=10$ & 250 & 270 & 300 & 290 & 280 & 240 & $\mathrm{R}$ & $\mathrm{T}$ \\
\hline Cover rate $\%$ & 60 & 75 & 55 & 70 & 85 & 75 & $\mathrm{E}$ & A \\
\hline Altitude in $\mathrm{m}$. & 460 & 450 & 660 & 550 & 600 & 630 & $\mathrm{~S}$ & $\mathrm{~T}$ \\
\hline Average height of veg. (m.) & 5 & 3.5 & 5 & 6 & 4 & 6 & $\mathrm{~N}$ & $\mathrm{U}$ \\
\hline Slope $\%$ & 20 & 30 & 15 & 25 & 25 & 15 & $\mathrm{C}$ & $\mathrm{S}$ \\
\hline Orientation & $\mathrm{E}$ & $\mathrm{E}$ & $\mathrm{N}$ & $\mathrm{W}$ & W & $\mathrm{N}$ & I & \\
\hline Order number & 1 & 2 & 3 & 4 & 5 & 6 & $\mathrm{a}$ & \\
\hline \multicolumn{9}{|l|}{ Characteristic of association and higher units } \\
\hline Quercus calliprinos Webb. & 4 & 3 & 2 & 1 & 1 & 2 & $\mathrm{~V}$ & $\mathrm{~N}$ \\
\hline Ceratonia siliqua $\mathrm{L}$. & 3 & 1 & 2 & 2 & & & IV & $\mathrm{N}$ \\
\hline Retama raetam (Forssk.) Webb \& Berthel. & 2 & 2 & & 1 & & & III & $\mathrm{N}$ \\
\hline Quercus look Kotschy & 2 & 1 & 1 & & & & IV & $\mathrm{E}$ \\
\hline Quercus coccifera L. & & 1 & & & & & $\mathrm{I}$ & $\mathrm{N}$ \\
\hline
\end{tabular}




\begin{tabular}{|c|c|c|c|c|c|c|c|c|}
\hline Species & & & & & & & & \\
\hline Pyrus syriaca Boiss. & & 1 & & & & & $\mathrm{I}$ & $\mathrm{E}$ \\
\hline Quercus inthaburensis L. & 1 & 1 & & 2 & & & III & $\mathrm{N}$ \\
\hline Quercus libani G. Olivier. & 1 & & 1 & & & 1 & III & $\mathrm{N}$ \\
\hline Companions & & & & & & & & \\
\hline Pistacia palaestina Boiss. & 2 & 2 & 2 & 1 & & & IV & $\mathrm{E}$ \\
\hline Pistacia atlantica Desf. & & & 1 & 1 & & & III & $\mathrm{N}$ \\
\hline Schinus molle L. & 2 & & 1 & & & 1 & III & $\mathrm{N}$ \\
\hline Pistacia saportae Burnat. & & & & 1 & & & I & $\mathrm{N}$ \\
\hline Rhus coriaria $\mathrm{L}$. & & & 1 & & & & I & $\mathrm{N}$ \\
\hline Pistacia lentiscus $L$. & I & & 1 & & I & & III & $\mathrm{N}$ \\
\hline Pinus halepensis Miller & 1 & & & & 1 & & III & $\mathrm{N}$ \\
\hline Pinus pinea $\mathrm{L}$. & & 1 & & 1 & & & II & $\mathrm{N}$ \\
\hline Pinus canariensis $\mathrm{C}$. Smith & & & & & & 1 & I & $\mathrm{N}$ \\
\hline Pinus brutia Tenore & & & & 1 & & 1 & II & $\mathrm{N}$ \\
\hline Cupressus sempervirens $\mathrm{L}$. var. horizontalis Miller & & 1 & & & & & I & $\mathrm{N}$ \\
\hline Pinus brutia Tenore & & 2 & & & & & I & $\mathrm{N}$ \\
\hline Cupressus arizonica Greene & & 1 & & & 1 & & II & $\mathrm{N}$ \\
\hline Cupressus macrocarpa Hartw. & & 1 & 1 & & 1 & & III & $\mathrm{N}$ \\
\hline Cupressus sempervirens L. & 1 & & & & & & I & $\mathrm{N}$ \\
\hline Thuja occidentalis L. & & & & & 1 & & I & $\mathrm{N}$ \\
\hline Zizyphus Spina-christi L. & 1 & & & & & & I & $\mathrm{N}$ \\
\hline Rhamnus palaestinus Boiss & 1 & & 1 & & & 1 & III & E \\
\hline Rhamnus alaternus L. & 1 & & 1 & & 1 & & III & $\mathrm{N}$ \\
\hline Zizyphus Lotus (L.) lam. & & & & & 1 & & I & $\mathrm{N}$ \\
\hline Rhamnus lycioides $\mathrm{L}$. & 1 & & & & & & I & $\mathrm{E}$ \\
\hline Crataegus oriana (L.) DC. & & 1 & & & & & I & $\mathrm{N}$ \\
\hline Crataegus azarolus L. & & & & & & & & $\mathrm{N}$ \\
\hline Amygdalus communis $\mathrm{L}$. & & 1 & & 1 & & & I & $\mathrm{N}$ \\
\hline Prunus dulcis (Miller) D. A. Webb. & & & & & & & I & $\mathrm{N}$ \\
\hline Sarcopoterium spinosum (L.) Spach & 2 & & & 1 & 1 & & III & $\mathrm{E}$ \\
\hline Acacia salicina Lindl. & & 1 & & & & & I & $\mathrm{N}$ \\
\hline Acacia cyanophylla Lindl. & & & & 1 & & & I & $\mathrm{N}$ \\
\hline Sophora japonica $\mathrm{L}$. & & & 1 & & & & I & $\mathrm{N}$ \\
\hline Calicotome villosa (Poir) Link. & 1 & & & & & & I & $\mathrm{N}$ \\
\hline Spartium junceum L. & & & & & 1 & & I & $\mathrm{N}$ \\
\hline Cersis siliquastrum L. & & & & & 1 & & I & $\mathrm{N}$ \\
\hline Poinciana gillesii Hook. & 1 & & & & & & I & $\mathrm{N}$ \\
\hline Acacia radiana Savi. & & & & & & & & $\mathrm{N}$ \\
\hline Olea europaea $\mathrm{L}$. & & & & & 1 & & I & $\mathrm{N}$ \\
\hline Phillyria media $\mathrm{L}$. & & & 1 & & 1 & & I & $\mathrm{N}$ \\
\hline Styrex officinalis $\mathrm{L}$. & & & & & & & I & $\mathrm{N}$ \\
\hline Laurus nobilis $\mathrm{L}$. & & 1 & & & & & I & $\mathrm{N}$ \\
\hline Balanites aegyptiaca (L.) Delile & & & & & 1 & & $\mathrm{I}$ & $\mathrm{N}$ \\
\hline Salix alba $\mathrm{L}$. & & & & & & & & $\mathrm{N}$ \\
\hline Populus alba $\mathrm{L}$. & & & & 1 & & & I & $\mathrm{N}$ \\
\hline Populus nigra $\mathrm{L}$. & & & & & 1 & & I & $\mathrm{N}$ \\
\hline Populus euphratica Oliv. & & & & & & & & $\mathrm{N}$ \\
\hline Arbatus andrachne $\mathrm{L}$. & & & 1 & & & & I & $\mathrm{E}$ \\
\hline Platanus orientalis $\mathrm{L}$. & 1 & & & & & & I & $\mathrm{N}$ \\
\hline Eucalyptus gomphosephala DC. & & 1 & & & & & I & $\mathrm{N}$ \\
\hline Eucalyptus camaldulensis Dehnh & & & & & 1 & & I & $\mathrm{N}$ \\
\hline Ailanthus glandulosa Desf. & & & & 1 & & & I & $\mathrm{N}$ \\
\hline Melia azedarach $\mathrm{L}$. & & & 1 & & & & I & $\mathrm{N}$ \\
\hline Acer obtusifolium $\mathrm{Sm}$. & & 1 & & & & & I & $\mathrm{E}$ \\
\hline Tamarix articulate Vahl. & & 1 & & & & & I & $\mathrm{N}$ \\
\hline Tamarix aphylla $\mathrm{L}$. & 1 & & & & & & I & $\mathrm{N}$ \\
\hline Tamarix jordanis Boiss. & & 1 & & & & & $\mathrm{I}$ & $\mathrm{E}$ \\
\hline Tamarix palestina Bertol. & & 1 & & & & & $\mathrm{I}$ & $\mathrm{E}$ \\
\hline Atriplex halimus L. & & & 1 & & & & I & $\mathrm{N}$ \\
\hline Ficus retusa $\mathrm{L}$. & & 1 & & & & & I & $\mathrm{N}$ \\
\hline Ficus sycomorus $\mathrm{L}$. & & & & & & 1 & $\mathrm{I}$ & $\mathrm{N}$ \\
\hline Ficus cariaca L. & 1 & & & & & & I & $\mathrm{E}$ \\
\hline Ficus benjamina $\mathrm{L}$. & & & & & 1 & & I & $\mathrm{N}$ \\
\hline Morus alba L. & & & & 1 & & & I & $\mathrm{N}$ \\
\hline Morus nigara $\mathrm{L}$. & & & & & & 1 & I & $\mathrm{N}$ \\
\hline Brachychiton populneus (Schott \& Endl.) R. Br. & & & & 1 & & & $\mathrm{I}$ & $\mathrm{N}$ \\
\hline Anagyris foetida $\mathrm{L}$. & & & 1 & & & & $\mathrm{I}$ & $\mathrm{N}$ \\
\hline
\end{tabular}




\begin{tabular}{|c|c|c|c|c|c|c|c|c|}
\hline Species & & & & & & & & \\
\hline Lycium shawii Reom. \& Schult. & & & 1 & & & & I & $\mathrm{E}$ \\
\hline Lycium barbarum L. & & 1 & & & & & I & $\mathrm{N}$ \\
\hline Lycium europaeum L. & 1 & & & 1 & & & I & $\mathrm{N}$ \\
\hline Artemisia sieberi Besser & & & & & 1 & & I & $\mathrm{N}$ \\
\hline Juglans regia $\mathrm{L}$. & & & & & 1 & & I & $\mathrm{N}$ \\
\hline Casuarina equesitifolia $\mathrm{L}$. & & & & 1 & & & I & $\mathrm{N}$ \\
\hline Celtis australis L. & & & & & & & & $\mathrm{N}$ \\
\hline Capparis spinosa L. & & 1 & & & & & I & $\mathrm{N}$ \\
\hline Phlomis brachyodon (Boiss.) Zohary & & & 1 & & & 1 & I & $\mathrm{E}$ \\
\hline Phlomis pungens Willd. & & & & 1 & & & I & $\mathrm{N}$ \\
\hline
\end{tabular}

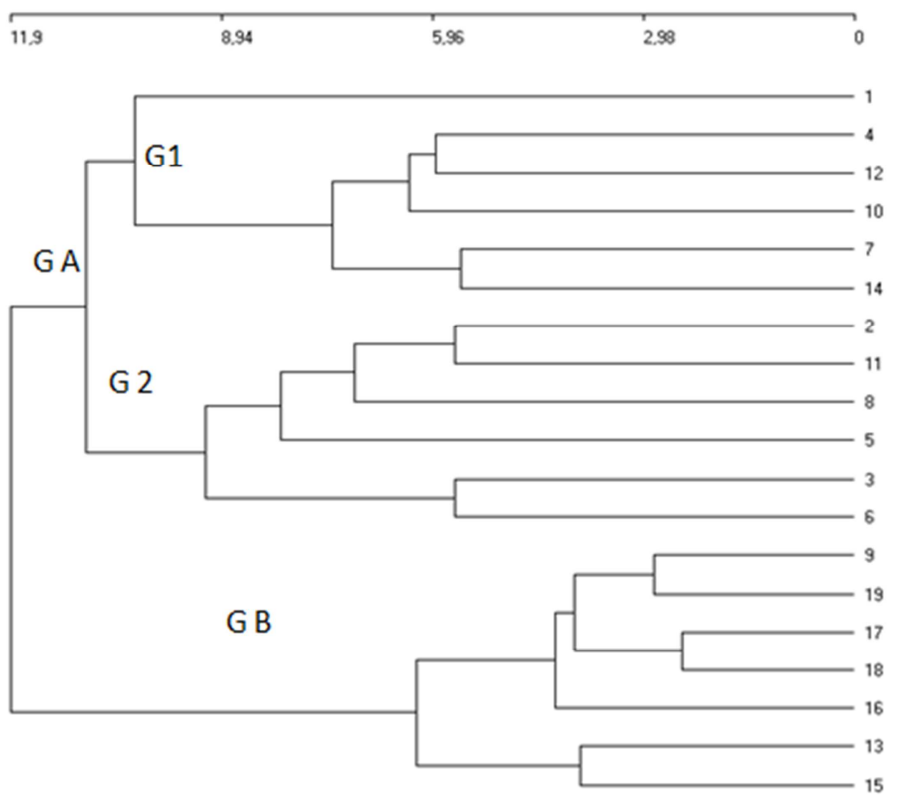

Figure 4. Cluster Analysis.

Table 4. Association 3: Pino halepensis-Cupressetum sempervirentis ass. nova.

\begin{tabular}{|c|c|c|c|c|c|c|c|c|c|}
\hline \multicolumn{10}{|l|}{ Species } \\
\hline Releve of cluster & 9 & 19 & 17 & 18 & 16 & 13 & 15 & $\mathrm{P}$ & $\mathrm{S}$ \\
\hline Surface in $\mathrm{m}^{2} 1=10$ & 330 & 350 & 600 & 570 & 530 & 580 & 490 & $\mathrm{R}$ & $\mathrm{T}$ \\
\hline Cover rate $\%$ & 70 & 60 & 80 & 70 & 65 & 75 & 85 & $\mathrm{E}$ & A \\
\hline Altitude in $\mathrm{m}$. & 580 & 460 & 520 & 550 & 700 & 490 & 750 & $\mathrm{~S}$ & $\mathrm{~T}$ \\
\hline Average height of veg. (m.) & 8 & 10 & 6 & 9 & 8 & 7 & 9 & $\mathrm{~N}$ & $\mathrm{U}$ \\
\hline Slope \% & 25 & 30 & 15 & 20 & 25 & 10 & 5 & $\mathrm{C}$ & $\mathrm{S}$ \\
\hline Orientation & W & $\mathrm{N}$ & $\mathrm{E}$ & $\mathrm{W}$ & W & $\mathrm{N}$ & $\mathrm{N}$ & I & \\
\hline \multicolumn{10}{|l|}{ Characteristic of association and higher units } \\
\hline Cupressus sempervirens L. & 5 & 2 & 2 & 3 & 1 & & & $\mathrm{~V}$ & $\mathrm{~N}$ \\
\hline Pinus halepensis Miller & 4 & 2 & 2 & 1 & 1 & & & IV & $\mathrm{N}$ \\
\hline Pinus Pinea L. & 2 & 2 & & & 1 & & 1 & IV & $\mathrm{N}$ \\
\hline Cupressus sempervirens L. var. horizontalis Miller & 2 & & & 1 & 1 & & & III & $\mathrm{N}$ \\
\hline Cupressus arizonica Greene & & & & 1 & & & 1 & I & $\mathrm{N}$ \\
\hline Thuja occidentalis L. & & 1 & & & & 1 & & I & $\mathrm{N}$ \\
\hline \multicolumn{10}{|l|}{ Companions } \\
\hline Pistacia palaestina Boiss. & 3 & & 2 & & 2 & & 1 & IV & $\mathrm{E}$ \\
\hline Pistacia atlantica Desf. & & & 2 & & 1 & & 1 & III & $\mathrm{N}$ \\
\hline Schinus molle L. & & 1 & & & & & 1 & I & $\mathrm{N}$ \\
\hline Pistacia saportae Burnat & & 1 & & 1 & 1 & 1 & & III & $\mathrm{N}$ \\
\hline Rhus coriaria $\mathrm{L}$. & 2 & & & 2 & & & 1 & III & $\mathrm{N}$ \\
\hline Pistacia lentiscus L. & & 1 & & 1 & & 1 & & III & $\mathrm{N}$ \\
\hline Pinus canariensis C. Smith & & 1 & & & & & & I & $\mathrm{N}$ \\
\hline Pinus brutia Tenore & & 1 & & & 2 & & & $\mathrm{I}$ & $\mathrm{N}$ \\
\hline
\end{tabular}




\begin{tabular}{|c|c|c|c|c|c|c|c|c|c|}
\hline Companions & & & & & & & & & \\
\hline Quercus look Kotschy & & 1 & & & 2 & & 2 & III & $\mathrm{E}$ \\
\hline Quercus calliprinos Webb. & 1 & & & & 2 & & & I & $\mathrm{N}$ \\
\hline Quercus coccifera $\mathrm{L}$. & & & & 1 & & 1 & & I & $\mathrm{N}$ \\
\hline Quercus inthaburensis L. & & & & 2 & & & 1 & II & $\mathrm{N}$ \\
\hline Quercus libani G. Olivier & & & & & 2 & & 1 & I & $\mathrm{N}$ \\
\hline Zizyphus Spina-christi L. & & & 1 & & & & & I & $\mathrm{N}$ \\
\hline Rhamnus palaestinus Boiss & 2 & & & & & 1 & & I & $\mathrm{E}$ \\
\hline Rhamnus alaternus $\mathrm{L}$. & & & 1 & & & 1 & & I & $\mathrm{N}$ \\
\hline Zizyphus Lotus (L) Lam. & & & & & 1 & & & I & $\mathrm{N}$ \\
\hline Rhamnus lycioides $\mathrm{L}$ & & & 1 & & 1 & 1 & & II & $\mathrm{E}$ \\
\hline Pyrus syriaca Boiss. & & & 1 & & & & 1 & I & E \\
\hline Crataegus oriana (L) DC. & & & & & & 1 & & I & $\mathrm{N}$ \\
\hline Crataegus azarolus L. & & & 1 & & & & & I & $\mathrm{N}$ \\
\hline Amygdalus communis $\mathrm{L}$. & & & & & 1 & & & I & $\mathrm{N}$ \\
\hline Prunus dulcis (Miller) D. A. Webb. & & 1 & 1 & & & 1 & & II & $\mathrm{N}$ \\
\hline Sarcopoterium spinosum (L.) Spach & 1 & & & 1 & & & & II & E \\
\hline Ceratonia siliqua $\mathrm{L}$. & 2 & & 1 & & 2 & & 1 & III & $\mathrm{N}$ \\
\hline Retama raetam (Forssk.) Webb \& Berthel. & & 1 & & & & & & I & $\mathrm{N}$ \\
\hline Acacia salicina Lindl. & 1 & & & & & & & I & $\mathrm{N}$ \\
\hline Acacia cyanophylla Lindl. & & & & & 1 & & & I & $\mathrm{N}$ \\
\hline Sophora japonica $\mathrm{L}$. & & 1 & & & & & & I & $\mathrm{N}$ \\
\hline Calicotome villosa (Poir) Link. & & & 1 & & & & & I & $\mathrm{N}$ \\
\hline Spartium junceum L. & & & & 1 & & & & I & $\mathrm{N}$ \\
\hline Cersis siliquastrum $\mathrm{L}$. & & 1 & & & & & & I & $\mathrm{N}$ \\
\hline Poinciana gillesii Hook. & & & & & & 1 & & I & $\mathrm{N}$ \\
\hline Acacia radiana Savi & & & & 1 & & & & I & $\mathrm{N}$ \\
\hline Olea europaea L. & & & & & 1 & & 1 & I & $\mathrm{N}$ \\
\hline Phillyria media $\mathrm{L}$. & & & 1 & & 1 & & & I & $\mathrm{N}$ \\
\hline Styrex officinalis $\mathrm{L}$. & & & & & 1 & & & I & $\mathrm{N}$ \\
\hline Laurus nobilis L. & & & 1 & & & & & I & $\mathrm{N}$ \\
\hline Balanites aegyptiaca (L.) Delile & & & & 2 & & & & I & $\mathrm{N}$ \\
\hline Salix alba $\mathrm{L}$. & & 1 & & & & & & I & $\mathrm{N}$ \\
\hline Populus alba L. & & & & 2 & & & & I & $\mathrm{N}$ \\
\hline Populus nigra $\mathrm{L}$. & & 1 & & & & & & I & $\mathrm{N}$ \\
\hline Populus euphratica Oliv. & & & 1 & & & & & I & $\mathrm{N}$ \\
\hline Arbatus andrachne $\mathrm{L}$. & & & & & 1 & & & I & $\mathrm{E}$ \\
\hline Platanus orientalis $\mathrm{L}$. & & & & & & 1 & & I & $\mathrm{N}$ \\
\hline Eucalyptus gomphosephala DC. & & & & & 1 & & & I & $\mathrm{N}$ \\
\hline Eucalyptus camaldulensis Dehnh & & & & 1 & & & & I & $\mathrm{N}$ \\
\hline Ailanthus glandulosa Desf.. & & & 1 & & & & & I & $\mathrm{N}$ \\
\hline Melia azedarach $\mathrm{L}$. & & & 1 & & & & & I & $\mathrm{N}$ \\
\hline Acer obtusifolium $\mathrm{Sm}$. & & & & 1 & & & & I & $\mathrm{E}$ \\
\hline Tamarix articulate Vahl & & & & & 1 & & & I & $\mathrm{N}$ \\
\hline Tamarix aphylla $\mathrm{L}$. & & & 1 & & & & & I & $\mathrm{N}$ \\
\hline Tamarix jordanis Boiss. & & & 1 & & & & & I & $\mathrm{E}$ \\
\hline Tamarix palestina Bertol. & & & 1 & & & & & I & $\mathrm{E}$ \\
\hline Atriplex halimus L. & & & 1 & & & & & I & $\mathrm{N}$ \\
\hline Ficus retusa $\mathrm{L}$. & 1 & & & & 2 & & & I & $\mathrm{N}$ \\
\hline Ficus sycomorus $\mathrm{L}$. & & & 1 & & 1 & & & I & $\mathrm{N}$ \\
\hline Ficus cariaca $\mathrm{L}$. & 1 & & & & & & & I & $\mathrm{N}$ \\
\hline Ficus benjamina $\mathrm{L}$. & 1 & & & & & & & I & $\mathrm{N}$ \\
\hline Morus alba $\mathrm{L}$. & & 1 & & & & & & I & $\mathrm{N}$ \\
\hline Morus nigara L. & & 1 & & 1 & & & & I & $\mathrm{N}$ \\
\hline Spartium junceum L. & & & & 2 & & & & I & $\mathrm{N}$ \\
\hline Anagyris foetida $\mathrm{L}$. & & & 1 & & & & & I & $\mathrm{N}$ \\
\hline Lycium shawii Reom. \& Schult.. & & & & & & 1 & & I & $\mathrm{E}$ \\
\hline Lycium barbarum $\mathrm{L}$. & & & 1 & & & & & I & $\mathrm{N}$ \\
\hline Lycium europaeum $\mathrm{L}$. & & 1 & & & & & & I & $\mathrm{N}$ \\
\hline Artemisia sieberi Besser & & 1 & & & & & & I & $\mathrm{N}$ \\
\hline Juglans regia L. & 1 & & & & & & & I & $\mathrm{N}$ \\
\hline Casuarina equesitifolia $\mathrm{L}$. & & & & 1 & & & & I & $\mathrm{N}$ \\
\hline Celtis australis $\mathrm{L}$. & & & 1 & & & & & I & $\mathrm{N}$ \\
\hline Jacaranda mimosaefolia D. Don & & & & 1 & & & & I & $\mathrm{N}$ \\
\hline Capparis spinosa $\mathrm{L}$. & 1 & & & & & & & I & $\mathrm{N}$ \\
\hline Brachychiton populneus (Schott \& Endl.) R. Br. & 1 & & & & & & & I & $\mathrm{N}$ \\
\hline Phlomis brachyodon (Boiss.) Zohary & & & & & + & & & & $\mathrm{E}$ \\
\hline Phlomis pungens Willd. & & & & & 1 & & & $\mathrm{I}$ & $\mathrm{N}$ \\
\hline
\end{tabular}


Table 5. Synesthetic representation of associations.

\begin{tabular}{|c|c|c|c|c|c|c|}
\hline \multicolumn{7}{|l|}{ Synesthetic representation } \\
\hline Species & ASL1 & ASL2 & ASL3 & Status & Life form & Family \\
\hline Quercus look Kotschy & $\mathrm{V}$ & IV & III & $\mathrm{E}$ & $\mathrm{T}$ & Fagaceae \\
\hline Pistacia lentiscus L. & Iv & III & III & $\mathrm{N}$ & Sh & Anacardiaceae \\
\hline Pistacia palaestina Boiss. & III & IV & IV & $\mathrm{E}$ & $\mathrm{T}$ & Anacardiaceae \\
\hline Quercus calliprinos Webb. & I & $\mathrm{V}$ & I & $\mathrm{N}$ & $\mathrm{T}$ & Fagaceae \\
\hline Quercus coccifera $\mathrm{L}$. & I & I & I & $\mathrm{N}$ & $\mathrm{T}$ & Fagaceae \\
\hline Pyrus syriac Boiss. & I & I & I & $\mathrm{E}$ & $\mathrm{T}$ & Rosaceae \\
\hline Quercus inthaburensis Decne. & III & III & II & $\mathrm{N}$ & $\mathrm{T}$ & Fagaceae \\
\hline Quercus libani G. Olivier & II & III & $\mathrm{I}$ & $\mathrm{N}$ & $\mathrm{T}$ & Fagaceae \\
\hline \multicolumn{7}{|l|}{ Companions } \\
\hline Rhamnus palaestinus Boiss. & $\mathrm{Iv}$ & III & I & $\mathrm{E}$ & Pha. & Rhamnaceae \\
\hline Rhamnus alaternus L. & I & III & I & $\mathrm{N}$ & $\mathrm{T}$ & Rhamnaceae \\
\hline Zizyphus Spina-christi L. & I & I & I & $\mathrm{N}$ & $\mathrm{T}$ & Rhamnaceae \\
\hline Zizyphus Lotus (L.) Lam. & I & I & I & $\mathrm{N}$ & Sh & Rhamnaceae \\
\hline Rhamnus lycioides $\mathrm{L}$. & I & I & II & $\mathrm{E}$ & Sh & Rhamnaceae \\
\hline Schinus molle L. & I & III & I & $\mathrm{N}$ & $\mathrm{T}$ & Anacardiaceae \\
\hline Pistacia saportae Burnat. & II & I & III & $\mathrm{N}$ & $\mathrm{T}$ & Anacardiaceae \\
\hline Rhus coriaria $\mathrm{L}$. & I & I & III & $\mathrm{N}$ & $\mathrm{T}$ & Anacardiaceae \\
\hline Pistacia atlantica Desf. & I & III & III & $\mathrm{N}$ & $\mathrm{T}$ & Anacardiaceae \\
\hline Pinus halepensis Miller & I & III & IV & $\mathrm{N}$ & $\mathrm{T}$ & Pinaceae \\
\hline Pinus Pinea L. & I & II & IV & $\mathrm{N}$ & $\mathrm{T}$ & Pinaceae \\
\hline Pinus canariensis C. Smith & I & I & I & $\mathrm{N}$ & $\mathrm{T}$ & Pinaceae \\
\hline Pinus brutia Tenore & I & II & I & $\mathrm{N}$ & $\mathrm{T}$ & Pinaceae \\
\hline Cupressus sempervirens $\mathrm{L}$. var. horizontalis Miller & I & I & III & $\mathrm{N}$ & $\mathrm{T}$ & Cupressaceae \\
\hline Cupressus arizonica Greene & I & II & I & $\mathrm{N}$ & $\mathrm{T}$ & Cupressaceae \\
\hline Thuja occidentalis L. & I & I & I & $\mathrm{N}$ & $\mathrm{T}$ & Cupressaceae \\
\hline Cupressus sempervirens $\mathrm{L}$. & I & I & $\mathrm{V}$ & $\mathrm{N}$ & $\mathrm{T}$ & Cupressaceae \\
\hline Crataegus oriana (L.) DC & & I & I & $\mathrm{N}$ & $\mathrm{T}$ & Rosaceae \\
\hline Crataegus azarolus $\mathrm{L}$. & & & I & $\mathrm{N}$ & $\mathrm{T}$ & Rosaceae \\
\hline Amygdalus communis L. & I & I & I & $\mathrm{N}$ & $\mathrm{T}$ & Rosaceae \\
\hline Prunus dulcis (Mill.) D. A. Webb & III & I & II & $\mathrm{N}$ & $\mathrm{T}$ & Rosaceae \\
\hline Sarcopoterium spinosum (L.) Spach & III & III & II & $\mathrm{E}$ & Cham & Rosaceae \\
\hline Ceratonia siliqua $\mathrm{L}$. & I & III & $\mathrm{V}$ & $\mathrm{N}$ & $\mathrm{T}$ & Fabaceae \\
\hline Retama raetam (Forssk.) Webb \& Berthel. & I & III & I & $\mathrm{N}$ & $\mathrm{T}$ & Fabaceae \\
\hline Acacia salicina Lindl. & I & I & I & $\mathrm{N}$ & $\mathrm{T}$ & Fabaceae \\
\hline Acacia cyanophylla Lindl. & I & I & I & $\mathrm{N}$ & $\mathrm{T}$ & Fabaceae \\
\hline Sophora japonica $\mathrm{L}$. & I & I & I & $\mathrm{N}$ & $\mathrm{T}$ & Fabaceae \\
\hline Calicotome villosa (Poir.) Link & I & I & I & $\mathrm{N}$ & Sh & Fabaceae \\
\hline Spartium junceum L. & I & I & I & $\mathrm{N}$ & Sh & Fabaceae \\
\hline Cersis siliquastrum $\mathrm{L}$. & II & I & I & $\mathrm{N}$ & $\mathrm{T}$ & Fabaceae \\
\hline Poinciana gillesii Hook. & I & I & I & $\mathrm{N}$ & Sh & Fabaceae \\
\hline Acacia radiana Savi. & & & I & $\mathrm{N}$ & $\mathrm{T}$ & Mimosaceae \\
\hline Olea europaea L. & I & I & I & $\mathrm{N}$ & $\mathrm{T}$ & Oleaceae \\
\hline Phillyria media $\mathrm{L}$. & II & I & I & $\mathrm{N}$ & $\mathrm{T}$ & Oleaceae \\
\hline Styrex officinalis L. & & I & I & $\mathrm{N}$ & $\mathrm{Sh}$ & Styracaceae \\
\hline Laurus nobilis L. & I & I & I & $\mathrm{N}$ & $\mathrm{T}$ & Lauraceae \\
\hline Balanites aegyptiaca (L.) Delile & II & I & I & $\mathrm{N}$ & $\mathrm{T}$ & Zygophyllaceae \\
\hline Salix alba $\mathrm{L}$. & & & I & $\mathrm{N}$ & $\mathrm{T}$ & Salicaceae \\
\hline Populus alba $\mathrm{L}$. & I & I & I & $\mathrm{N}$ & $\mathrm{T}$ & Salicaceae \\
\hline Populus nigra $\mathrm{L}$. & I & I & I & $\mathrm{N}$ & $\mathrm{T}$ & Salicaceae \\
\hline Populus euphratica Oliv. & & & I & $\mathrm{N}$ & $\mathrm{T}$ & Salicaceae \\
\hline Arbatus andrachne $\mathrm{L}$. & I & I & I & $\mathrm{E}$ & $\mathrm{T}$ & Ericaceae \\
\hline Platanus orientalis L. & & I & I & $\mathrm{N}$ & $\mathrm{T}$ & Platanaceae \\
\hline Eucalyptus gomphosephala DC. & & I & I & $\mathrm{N}$ & $\mathrm{T}$ & Myrtaceae \\
\hline Eucalyptus camaldulensis Dehnh & I & I & I & $\mathrm{N}$ & $\mathrm{T}$ & Myrtaceae \\
\hline Ailanthus glandulosa Desf. & I & I & I & $\mathrm{N}$ & $\mathrm{T}$ & Simaroubiaceae \\
\hline Melia azedarach L. & I & I & I & $\mathrm{N}$ & $\mathrm{T}$ & Meliaceae \\
\hline Acer obtusifolium $\mathrm{Sm}$. & I & I & I & $\mathrm{E}$ & $\mathrm{T}$ & Aceracea \\
\hline Tamarix articulate Vahl. & I & I & I & $\mathrm{N}$ & $\mathrm{T}$ & Tamaricaceae \\
\hline Tamarix aphylla $\mathrm{L}$. & I & I & I & $\mathrm{N}$ & $\mathrm{T}$ & Tamaricaceae \\
\hline Tamarix jordanis Boiss. & I & I & I & $\mathrm{E}$ & $\mathrm{T}$ & Tamaricaceae \\
\hline Tamarix palestina Bertol. & I & I & I & E & $\mathrm{T}$ & Tamaricaceae \\
\hline Atriplex halimus $\mathrm{L}$. & I & I & I & $\mathrm{N}$ & Sh & Amaranthaceae \\
\hline Ficus retusa L. & I & I & I & $\mathrm{N}$ & $\mathrm{T}$ & Moraceae \\
\hline
\end{tabular}




\begin{tabular}{|c|c|c|c|c|c|c|}
\hline \multicolumn{7}{|l|}{ Companions } \\
\hline Ficus sycomorus L. & II & I & $\mathrm{I}$ & $\mathrm{N}$ & $\mathrm{T}$ & Moraceae \\
\hline Ficus cariaca L. & II & I & I & $\mathrm{E}$ & $\mathrm{T}$ & Moraceae \\
\hline Ficus benjamina $\mathrm{L}$. & I & I & I & $\mathrm{N}$ & $\mathrm{T}$ & Moraceae \\
\hline Morus alba $\mathrm{L}$. & I & I & I & $\mathrm{N}$ & $\mathrm{T}$ & Moraceae \\
\hline Morus nigara L. & II & I & I & $\mathrm{N}$ & $\mathrm{T}$ & Moraceae \\
\hline Hypecoum pendulum $\mathrm{L}$. & & I & I & $\mathrm{E}$ & Annual & Papaveraceae \\
\hline Anagyris foetida $\mathrm{L}$. & I & I & I & $\mathrm{N}$ & Phan & Papilionaceace \\
\hline Lycium barbarum $\mathrm{L}$. & I & I & I & $\mathrm{N}$ & Sh & Solanaceae \\
\hline Lycium europaeum L. & & I & I & $\mathrm{N}$ & Sh & Solanaceae \\
\hline Artemisia sieberi Besser & I & I & I & $\mathrm{N}$ & cham & Asteraceae \\
\hline Juglans regia $\mathrm{L}$. & I & I & I & $\mathrm{N}$ & $\mathrm{T}$ & Juglandaceae \\
\hline Casuarina equesitifolia $\mathrm{L}$. & I & I & I & $\mathrm{N}$ & $\mathrm{T}$ & Casuarenaceae \\
\hline Celtis australis L. & I & & I & $\mathrm{N}$ & $\mathrm{T}$ & Ulmaceae \\
\hline Jacaranda mimosaefolia D. Don & I & I & I & $\mathrm{N}$ & $\mathrm{T}$ & Bignoniaceae \\
\hline Phlomis brachyodon (Boiss.) Zohary & I & I & I & $\mathrm{E}$ & Sh & Lamiaceae \\
\hline Phlomis chrysophylla Boiss. & I & I & I & $\mathrm{E}$ & cham & Lamiaceae \\
\hline Phlomis pungens Willd & $\mathrm{I}$ & $\mathrm{I}$ & I & $\mathrm{N}$ & Sh & Lamiaceae \\
\hline
\end{tabular}

Percentage of plant species presence in the samples studies and communities: $\mathrm{V}=100 \%$, IV $=60.1 \%-80 \%$, III $=40.1 \%$ $60 \%, \mathrm{II}=20.1 \%-40 \%$ and $\mathrm{I}=0.1 \%-20 \%$. N: Native and $\mathrm{E}$ : Endemic, Sh: Shrub. Association (ASL), GI: Group one, G II: Group two, GIII: Group three. ASL1: Pistacio lentisciQuercetum lokii, ASL2: Ceratonio siliquae-Quercetum callipinii and ASL3: Pino halepensis-Curessetum sempervirentis.

Finally, in the first association, the high proportion of Pistacia lentiscus L., Pistacia palestina Boiss., and Rhamnus palestina Boiss, Rhamnus lycioides., Rhamnus alaternus L., allows us to include the communities in dry-xeric shrublands from Mediterranean region and centre of Asia minor in the order Pistachio lentisci -Rhamnetalia alaterni Rivas-Martínez, S., 1975 [42] in the alliance of the Oleo sylvestris-Ceratonion siliquae Br.-B1. ex Guinochet \& Drouineau 1944 [43, 44], and the forests adapting in dry to sub-humid environments as Rhamnus spp. and Quercus spp. in the order Rhamno lycioidisQuercion cocciferae Rivas Goday ex Rivas-Martinez 1975 [42], class of the Quercetalia calliprini Zohary 1955 [45] and Quercetalia ilicis Br.-Bl. ex Molinier [46-48]. Furthermore, in the second community, Sclerophyllous oaks, conifer forests and macchia with the high frequency of Ceratonia siliqua L. and Quercus look Kotschy, Quercus calliprinos Webb., Quercus coccifera L. and others species at arid, dry, sub-humid and humid area and $\mathrm{n}$ the thermo- to supramediterranean belts of the Eastern Mediterranean or Asia minor, as the same in alliance, the order and class of the first association. In the last association, we obtained high frequency of Pinus halepensis Miller, Pinus Pinea L., Cupressus sempervirens L., Cupressus macrocarpaa L., Cupressus arizonica Greene species in dry, subhumid and humid region and thermo-mesomediterranean in order Pinetalia halepensis Biondi et al. (2014) [49], class of the Quercetalia calliprini Zohary 1955 [45], and Quercetalia ilicis Br.-Bl. ex Molinier [46-48].

Syntaxonomical showing of these associations is:

Quercetalia ilicis Br.-B1. ex Molinier 1934, Quercetalia calliprini Zohary 1955.

Rhamno lycioidis-Quercion cocciferae Rivas Goday ex
Rivas-Martinez 1975

Pistachio lentisci -Rhamnetalia alaterni Rivas-Martínez 1975

1. Pistacio lentisci-Quercetum lokii Ighbareyeh J. M. H., A. A. Suliemieh, A. Cano-Ortiz \& E. Cano nova. hoc loco.

Rhamno lycioidis-Quercion cocciferae Rivas Goday ex Rivas-Martinez 1975

Quercetalia calliprini Zohary 1955

2. Ceratonio siliquae-Quercetum callipinii ass. nova.

Quercetalia ilicis Br.-Bl. ex Molinier 1934

Pinetalia halepensis Biondi et al. (2014)

3. Pino halepensis-Cupressetum sempervirentis ass. nova.

\section{Conclusion}

Despite the fact that more than a century and a half ago the establishment of the Wadi Al-Quf Forest Reserve, since the Ottoman era in the middle of the eighteenth century, but this Forest was able to preserve its environment and biodiversity, where it found more than 82 species of plants, including 16 plants a endemic rare. Wadi Al-Quf Forest Reserve belongs to Infra-thermomediterranean with a little Mesomediterranean thermotype and dry to sub-humid ombrotype. Moreover, during our study, we have three associations limited as Pistacio lentisci-Quercetum lokii Ighbareyeh M. H. J, A. A. Suliemieh, A. Cano-Ortiz \& E. Cano nova. hoc loco., Ceratonio siliquae-Quercetum callipinii ass. nova. and Pino halepensis-Curessetum sempervirentis ass. nova., with three alliance as PistachioQuercion lokii, Ceratonia siliquae-Quercion calliprinae and Pino halepensis-Cupression sempervirentis

In recent years, especially in the areas surrounding the study in the Hebron area as Idna Village, we were indicted eight new plant associations [5, 21, 22]:

Pistacio palaestinae-Quercetum Lokii*

Capparido sinaicae-Ceratonietum siliquae

Cerasus microcarpae-Quercetum ithaburensis*

Pyro siriacae-Abietetum cilicicae*

Abio ciliciae-Ceratonietum siliquae 
Periploco aphylli-Pinetum halepensis

Cytisopsis pseudocytiso-Tamaricetum tetragynae

Crataego sinaicae-Tamaricetum jordanii.

Whereas, in the last study 2018 by Ighbareyeh [21, 22], we describe three new plant associations:

Pino halepinsis -Quercetum lookii *.

Pistacio paletinae -Ceratonietum siliquae *.

Quercus libanii -Tamaricetum palestineae *.

* Associations in which olive cultivation is possible.

\section{References}

[1] Ighbareyeh, J. M. H., Cano-Ortiz, A. and Cano, E. 2014a. Biological and Bioclimatic Basis to Optimize Plant Production: Increased Economic Areas of Palestine. Agricultural Science Research Journal, 4, 10-20.

[2] Ighbareyeh, J. M. H., Cano-Ortiz, A., Cano Carmona, E., Suliemieh, A. A. A. and Ighbareyeh, M. M. H. 2017a. Flora Endemic Rare and Bioclimate of Palestine. Open Access Library Journal, 4, e3977. https://doi.org/10.4236/oalib.1103977.

[3] Ighbareyeh, J. M. H., Cano-Ortiz, A., Suliemieh, A. A. A., Ighbareyeh, M. M. H. and Cano, E. 2014c. Phytosociology with Other Characteristic Biologically and Ecologically of Plant in Palestine. American Journal of Plant Sciences, 5, 3104-3118. https://doi.org/10.4236/ajps.2014.520327.

[4] Ighbareyeh J. M. H., and Cano Carmona E. 2018a. A Phytosociological of Plant Communities and Biodiversity in the East-South of Idna Village- Hebron of Palestine. International Journal of Geosciences, 9, 44-58. https://doi.org/10.4236/ijg.2018.91003.

[5] Ighbareyeh J. M. H., Cano-Ortiz A. and Cano E., 2014. Biological resources management in Palestine, Department of Animal and Plant Biology and Ecology, Faculty of Experimental Sciences, University of Jaen, Jaen, Spain, P 100-105, doctorate thesis.

[6] Zohary M., 1962. Plant Life of Palestine. Ronald Press Company, New York.

[7] Shmida A., 1995. General References on Biodiversity and Theory of Ecological Richness Especially Concerned with Arid and Mediterranean Ecosystems, The Hebrew University, and Jerusalem.

[8] Médail F. and Quézel P., 1997. Hot-Spots Analysis for conservation of Plant Biodiversity in the Mediterranean Basin. Annals of the Missouri Botanical Garden, 84, 112-127. http://dx.doi.org/10.2307/2399957.

[9] Myers N. and Cowling R., 1999. Mediterranean Basin. In: Mittermeier, R. A., Myers, N. and Goettsch Mittermeier, C. Eds., Hotspots Earth's Biologically Richest and Most Endangered Terrestrial Ecoregions, CEMEX \& Conservation International, Mexico City, 254-267.

[10] Ighbareyeh J. M. H., Cano-Ortiz A., Suliemieh A. A. A., Ighbareyeh M. M. H. and Cano E., 2015a Assessing Crop Yield Sustainability under the Climatic and Bioclimatic Change in the Area of Palestine. American Journal of Climate Change, 4, 48-56. https://doi.org/10.4236/ajcc.2015.41005.

[11] Ighbareyeh J. M. H., Cano-Ortiz A., Suliemieh A. A. A.,
Ighbareyeh M. M. H. and Cano E., 2015b Assessment of Biology and Bioclimatology of Plant to Increase Economic in Palestine. International Journal of Research Studies in Biosciences, 3, 1-8.

[12] Danin A., 2004. Distribution Altas of Plants in Flora Palestine Area. 2nd Edition, Academy of Science and Humanities, Jerusalem, 520 .

[13] Ighbareyeh J. M. H., Cano-Ortiz A., Suliemieh A. A. A., Ighbareyeh M. M. H. and Cano E, 2015c. Biology and Bioclimatology Applied on Plant in Palestine. International Journal of Research Studies in Biosciences, 3, 79-86.

[14] Ighbareyeh J. M. H., Cano-Ortiz A., Suliemieh A. A. A., Ighbareyeh, M. M. H. and Cano E., 2015d. Study Effect of Biology and Bioclimatology Applied on Plant in the Area of Hebron at the South of Palestine. International Journal of Research Studies in Biosciences, 3, 56-64.

[15] Ighbareyeh Jehad M. H. et. al, 2016a. Study of Biology and Bioclimatology Applied of Apricot (Prunus Armeniaca L.): to Increase the Economy and Maintaining Food Security in Palestine International Journal of Research Studies in Biosciences 4 (2): 25-33. doi: dx.doi.org/10.20431/23490365.0402004 .

[16] Ighbareyeh J. M. H., Cano-Ortiz A., Suliemieh A. A. A., Ighbareyeh M. M. H. and Cano E., 2016b. Modeling of biology and bioclimatology applied studies on plant in Palestine. International Journal of Development Research, 6, 9585-9590. http://www.journalijdr.com.

[17] Ighbareyeh J. M. H., Cano-Ortiz A., Suliemieh A. A. A., Ighbareyeh M. M. H. and Cano E., 2015e. Effect of Biology and Bioclimatology Applied on Plant in the Area of Jenin at the North of Palestine. International Journal of Research Studies in Bioscience, 3, 1-6.

[18] Ighbareyeh J. M. H., Cano-Ortiz A., Cano E., Ighbareyeh M. M. H., Suliemieh A. A. A., 2016. Effect of Biology, Climatic and Bioclimatic Applied Studies on Plant: to Increase the Economy and Maintaining Food Security in the Jerusalem Occupied of Palestine. International Journal of Research Studies in Biosciences, 4, 54-60. http://www.arcjournals.org.

[19] Ighbareyeh J. M. H., Cano-Ortiz A., Suliemieh A. A. A., Ighbareyeh M. M. H., Cano E. and Hijjeh S., 2017c. Effect of Bioclimate Factors on Olive (Olea europea L.) Yield: To Increase the Economy and Maintaining Food Security in Palestine. International Journal of Development Research, 6, 10648-10652. https://doi.org/10.4236/oalib.1104119.

[20] Ighbareyeh J. M. H. and Cano Carmona E., 2018a A Phytosociological of Plant Communities and Biodiversity in the East-South of Idna Village- Hebron of Palestine. International Journal of Geosciences, 9, 44-58. https://doi.org/10.4236/ijg.2018.91003.

[21] Ighbareyeh J. M. H. and Carmona E. C., 2018b. Impact of Environment Conditions on Grapevine (Vitis vinifera L.): To Optimal Production and Sustainability, Achieving Food Security and Increasing the Palestinian Economy. Journal of Geoscience and Environment Protection, 6, 62-73. https://doi.org/10.4236/gep.2018.62005.

[22] Ighbareyeh Jehad M. H. et al., 2018c. Grapevine (Vitis vinifera L.) Yield and Climate Conditions of Palestine. Current Science, [S.1.], v. 114, n. Issue 8, mar. 2018. ISSN 0011-3891.

http://www.currentscience.org/index.php/CS/article/view/285. 
[23] Ighbareyeh J. M. H., Carmona E. C., Ortiz A. C. et al, 2018d. Analysis of physical factors of climate and bioclimate and their effects on almonds production to increase the economy in Hebron area of Palestine Arab J Geosci (2018) 11: 683. https://doi.org/10.1007/s12517-018-4026-0. Springer.

[24] Ighbareyeh J. M. H. et al., 2018e Impact of bioclimatic and climatic factors on Ficus carica L. yield: increasing the economy and maintaining the food security of Jerusalem in Palestine. Transylvanian Review: Vol XXVI, No. 34, P. 8719, November 2018 . http://transylvanianreviewjournal.org/index.php/TR/article/vie $\mathrm{w} / 3235$.

[25] Cano E., Musarella C., Piñar Fuentes J. C. \& Pinto-Gomes C., 2016. Vegetation series as a basis for habitats and species conservation: methodological aspects. Botanique 1:21.26.

[26] Pieroni A., Nebel S., Quave C., Munz H., Heinrich M., 2002. Ethnopharmacology of Liakra: traditional weedy vegetables of the Arbereshe of the Vulture area in Southern Italy. J Ethnopharmacol. 2002; 81: 165-185.

[27] Pieroni A., Nebel S., Santoro RF., Heinrich M., 2005. Food for two seasons: Culinary uses of non-cultivated local vegetables and mushrooms in a south Italian village. Int $\mathrm{J}$ Food Sci Nutr. 56: 245-272.

[28] Bonet M A., Vallès J. 2002. Use of non-crop food vascular plants in Montseny biosphere reserve (Catalonia, Iberian Peninsula) Int J Food Sci Nutr. 53: 225-248.

[29] Tardío J, Pardo-de Santayana M., Morales R., 2006. Ethnobotanical review of wild edible plants in Spain. Botanical Journal of the Linnean Society. 2006; 152: 27-71.

[30] Silva Joan B., Sfair Julia C., dos Santos, Nivea D., Porto Katia C., 2017. Different trait arrangements can blur the significance of ecological drivers of community assembly of mosses from rocky outcrops. Flora http://dx.doi.org/10.1016/j.flora.02.003.

[31] Braun-Blanquet J., 1979. Fitosociología. Bases para el estudio de las comunidades vegetales. Blume, Madrid, 820 p.

[32] Van Der Maarel E., 1979. Transformation of CoverAbundance Values in Phytosociology and Its Effects on Community Similarity. Vegetatio, 39, 97-114.

[33] Shaltout KH., El-Kady HF., Al-Sodany YM., 1995. Vegetation analysis of the Mediterranean region of Nile Delta. Vegetatio 116: $73-83$.

[34] Shaltout KH., Sheded MG., El-Kady HF., Al-Sodany YM., 2003. Phytosociology and size structure of Nitraria retusa along the Egyptian Red Sea coast. J. Arid Env. 53: 331-345.

[35] Morteza Djamalia, Simon Brewerb, Siegmar W. Brecklec, Stephen T. Jackson., 2012. Climatic determinism in phytogeographic regionalization a test from the IranoTuranian region SW and Central Asia. Flora 207 (2012) 237249.
[36] Biondi E., 2011. Phytosociology Today: Methodological and Conceptual Evolution. Plant Biosystems, 145, 19-29. https://doi.org/10.1080/11263504.2011.602748 https://doi.org/10.1007/BF00052021.

[37] Pott R., 2011. Phytosociology: A Modern Geobotanical Method. Plant Biosystems, 145, 9-18. https://doi.org/10.1080/11263504.2011.602740.

[38] Rivas-Martínez S., 1996. Clasificación bioclimática de la Tierra. Folia Bot. Matritensis. 16: 1-20.

[39] Rivas Martinez S., Sanchez Mata D. and Costa M., 1999. North American boreal and western temperate forest vegetation (Syntaxonomical synopsis of the potential natural plant communities of North America, II). Itinera Geobot. 12: 5-316.

[40] Rivas-Martınez S., Rivas Saenz S. and Penas A., 2011. Worldwide Bioclimatic Classification System. Global Geobot., 1, 1-634.

[41] Liogier A. H., 1996. La Flora de la Española. Vol. I-IX. Jardín Botánico Nacional Dr. Rafael Ma. Moscoso, ISBN, 84-8400217-9, Santo Domingo.

[42] Rivas-Martínez S. 1975. La vegetación de la clase Quercetea ilicis en España y Portugal. Anales Instituto Botánico Cavanilles, 31, 205-262.

[43] Guinochet, M. \& G. Drouineau, 1944. Notes sur la végétation et les sols aux environs d'Antibes (Alpes-Maritimes). Rec. Trav. Bot., 1: 22-40.

[44] Bardat J., Bioret F., Botineau M., Boullet V., Delpech R., Géhu J. M. et al., 2004. Prodrome des végétations de France. Coll. Patrimoines naturels 61. Muséum national d'histoire naturelle, Paris, $171 \mathrm{p}$.

[45] Zohary M., 1955, Geobotany. Merhavia (in Hebrew).

[46] Molinier R. 1934. Études phytosociologiques et écologiques en Provence occidentale. Anales Museo Historia Natural Marseille, 27, 1-273.

[47] Molinier R. 1968. Le dynamisme de la végétation provençale. Collectanea Botánica, 7, 817-844.

[48] Rivas-Martínez S., Díaz T. E., Fernández-González F., Izco J., Loidi J., Lousa M. and Penas E. 2002. Vascular Plant Communities of Spain and Portugal. Itinera Geobotanica, 15, 433-922.

[49] Biondi E., Blasi C., AllegrezzaM., Anzellotti I., Azzella M. M., Carli E., Casavecchia S., Copiz R., Del Vico E., Facioni L., Galdenzi D., Gasparri R., Lasen C., Pesaresi S., Poldini L., Sburlino G., Taffetani F., Vagge I., Zitti S. \& Zivkovic L. 2014. Plant communities of Italy: The Vegetation Prodrome. Plant Biosyst. 148: 728-814. 